\title{
Degradation of naturally produced hydroxylated polybrominated diphenyl ethers in Baltic Sea sediment via reductive debromination
}

\author{
Dennis Lindqvist $^{1}$ (D) Johan Gustafsson ${ }^{1}$ \\ Received: 8 May 2020 / Accepted: 11 January 2021 / Published online: 21 January 2021 \\ (C) The Author(s) 2021
}

\begin{abstract}
Over the last two decades, the occurrence of hydroxylated polybrominated diphenyl ethers (OH-PBDEs) has been observed to be nearly ubiquitous among Baltic Sea filamentous macroalgae. High concentrations are continuously recorded among red, green, and brown filamentous algae. Several of these algae species are ephemeral, and when large parts of the colonies decay at the end of their lifecycles, the OH-PBDEs are expected to largely partition to the sediment. In this study, the fate of OH-PBDEs in Baltic Sea sediment was investigated, with focus on the effect of reductive debromination. During chemical debromination, it was observed that the half-life could differ with as much as two orders of magnitude between a pentabrominated and a tetrabrominated congener. Using collected Baltic Sea sediment, it was further observed that the half-life of spiked pentabrominated OH-PBDEs spanned from a few days up to a few weeks in room temperature. At $4{ }^{\circ} \mathrm{C}$, it took 6 months to achieve a 50\% decrease in concentration of the fasted degrading congener. Clear differences in selectivity between chemical debromination and debromination in sediment were also observed when studying the major reaction products. Baltic Sea sediment seems to have a good capacity for reducing naturally produced OH-PBDEs.
\end{abstract}

Keywords Reduction $\cdot$ OH-PBDE $\cdot$ PBDE $\cdot$ Biodegradation $\cdot$ Algae $\cdot$ Sediment

\section{Introduction}

Many halogenated organic compounds are known to be naturally produced in both the terrestrial and marine environments, by, for example, algae, fungi, sponges, and bacteria (Gribble 2010). In the Baltic Sea, brominated substance such as bromophenols, hydroxylated polybrominated diphenyl ethers (OH-PBDEs), methoxylated PBDEs (MeO-PBDEs), and polybrominated dibenzo- $p$-dioxins have been identified in several species of filamentous macroalgae (Löfstrand et al. 2011). During summer in the Baltic Sea, the biomass of several species of filamentous macroalgae increases (HELCOM 2009). Furthermore, a large increase in the production of $\mathrm{OH}-$ PBDEs has also been observed during the summer, in the red algae Ceramium tenuicorne (Dahlgren et al. 2015). The

Responsible Editor: Roland Peter Kallenborn

Dennis Lindquist

dennis.lindqvist@aces.su.se

1 Department of Environmental science, Stockholm University, SE-106 91 Stockholm, Sweden increased biomass of filamentous macroalgae together with increased production of OH-PBDEs within these algae leads to a large input of OH-PBDEs to the Baltic Sea during summer. The large input in turn results in an increased exposure to OH-PBDEs among Baltic wildlife, as observed in, for example, blue mussels (Löfstrand et al. 2011). The concentration of these compounds in blue mussels has been observed to be much larger than the concentration of anthropogenic brominated pollutants, specifically the brominated flame retardant BDE47 (Löfstrand et al. 2011). Exposure to OH-PBDEs in wildlife may also stem from accumulation of algae produced MeO-PBDEs and a subsequent metabolic demethylation (Wan et al. 2009).

Upon death and decomposition of OH-PBDE-producing algae in the Baltic Sea, large quantities of OH-PBDEs are expected to be transferred to the sediment. High sorption to sediment has been reported for some chlorinated phenols (Isaacson and Frink 1984). Sorption to sediment does not only occur in the less polar protonated form but also in the ionic form (Schellenberg et al. 1984), which is important for OHPBDEs that generally have $\mathrm{p} K_{a}$ values close to or below the $\mathrm{pH}$ of seawater. Thus, sediment is expected to be the final sink for a large proportion of the naturally produced brominated 
compounds in the Baltic Sea. Based on the high concentrations of OH-PBDEs detected in C. tenuicorne (Dahlgren et al. 2015; Lindqvist et al. 2017) as well as in other macroalgae in the Baltic Sea (Löfstrand 2011), the annual input of OHPBDEs to Baltic Sea sediment is expected to be large.

The structurally related compound decabromodiphenyl ether (BDE209) has previously been shown to undergo reductive debromination in anaerobic sediment, and a large variety of lesser brominated PBDEs were observed to be produced (Tokarz et al. 2008). When Zhang and co-workers (2012) studied the transformation of 6-OH-BDE47 in Chinese sediment, a methylation to 6-MeO-BDE47 was observed.

The present study was divided into two parts. The first part was aimed to assess the relative chemical stability of the most abundant naturally produced $\mathrm{OH}-\mathrm{PBDE}$ congeners in the Baltic Sea towards reductive debromination, while simultaneously creating a map over degradation products formed by each congener. The second part was aimed to investigate the degradation of OH-PBDEs in collected Baltic Sea sediment and the role reductive debromination has in any observed degradation.

\section{Materials and methods}

\section{Chemicals and reagents}

All solvents used were of analytical grade quality and purchased from established brands. Metallic zinc powder was purchased from Sigma-Aldrich (Steinheim, Germany). All $\mathrm{OH}-\mathrm{PBDEs}$ and $\mathrm{MeO}-\mathrm{PBDEs}$ used for degradation tests and as reference compounds for sediment analysis were synthesized in house by Marsh and co-workers (2003). BDE99 and BDE139 were purchased from Wellington Laboratories (Guelph, ON, Canada). Diazomethane used for derivatization of OH-PBDEs was synthesized in house from $\mathrm{N}$-methyl- $\mathrm{N}$ nitroso- $p$-toulenesulfonamide (Diazald) purchased from Sigma-Aldrich (Steinheim, Germany).

\section{Samples}

Sediment samples were collected in late June outside of Askö, Sweden (Lat. 58.8215, Long. 17.6305), using an Ekman grab sampler and were stored at $4{ }^{\circ} \mathrm{C}$; seawater was collected simultaneously. Macroscopic objects such as algae and mussels were removed by hand from the sediment before centrifugation, decanting of the water, and mixing of the sediment by hand. The water sample was filtered through a filter paper followed by a $0.22-\mu \mathrm{m}$ membrane filter (Millipore, Burlington, MA, USA). The experiments were started the day after the samples were collected.

\section{Chemical reductive debromination}

Chemical debromination was conducted in a similar manner as one of the methods presented for reduction of 2,4,6tribromophenol by Tashiro et al. (1976). Nine OH-PBDEs were degraded, including one hexabrominated congener: 6OH-BDE137; four pentabrominated congeners: 6-OHBDE85, 2-OH-BDE123, 6-OH-BDE90, and 6-OH-BDE99; and four tetrabrominated congeners: 2'-OH-BDE66, 2'-OHBDE68, 6'-OH-BDE49, and 6-OH-BDE47. Two neutral pentabrominated compounds were also included in the experiment: 6-MeO-BDE99 and BDE99. Each test substance $(1 \mu \mathrm{g})$ dissolved in ethanol $(1.2 \mathrm{~mL})$ was added to a $2-\mathrm{mL}$ Eppendorf tube containing zinc powder $(50 \mathrm{mg})$. Hydrochloric acid $(\mathrm{HCl} ; 1.5 \mathrm{M}, 0.3 \mathrm{~mL})$ was added, and the sample was incubated at $50^{\circ} \mathrm{C}$ on an Eppendorf Thermomixer comfort (Eppendorf, Hamburg, Germany) mixing at $750 \mathrm{rpm}$. All compounds were tested individually in triplicates. Fifty microliters of the test solution was taken out at different time points and was transferred to a new Eppendorf tube containing water $(100 \mu \mathrm{L})$ and either pentane/diethyl ether $(9: 1,1.2 \mathrm{~mL})$ for OH-PBDEs or 2,2,4-trimethylpentane (TMP; $0.7 \mathrm{~mL}$ ) for PBDEs and MeO-PBDEs. The samples were mixed and the organic phase isolated. Neutral compounds were analyzed directly on gas chromatography mass spectrometry (GC-MS) whiles the OH-PBDEs were methylated using ethereal diazomethane $(300 \mu \mathrm{L})$ for $1.5 \mathrm{~h}$ at ambient temperature in darkness. After methylation, the samples were evaporated to dryness and resolved in TMP $(0.7 \mathrm{~mL})$ before analysis.

For the hexabrominated 6-OH-BDE137, aliquot samples were taken after $0,1,3,6,10$, and $15 \mathrm{~min}$. For pentabrominated OH-PBDEs and MeO-BDE99, aliquot samples were taken after 0, 2, 5, 9, 14, and $20 \mathrm{~min}$. For BDE99, aliquots were taken at $0,5,15,30$, and $60 \mathrm{~min}$. For 2'-OHBDE66 and 2'-OH-BDE68, aliquots were taken at 0,15, 45, 90 , and $150 \mathrm{~min}$. Lastly, for 6-OH-BDE47 and 6'-OHBDE49, aliquot samples were taken at $0,45,90,180$, and $240 \mathrm{~min}$. The variation in sampling time points depended on the varying reaction speed of each congener. Zinc and $\mathrm{HCl}$ were used in excess to achieve a pseudo first-order reaction. However, after $1 \mathrm{~h}$, the reaction started to become limited by the acid. Hence, an additional drop of $\mathrm{HCl}(6 \mathrm{M})$ was added to maintain first-order kinetics when aliquot samples were taken out for the tetrabrominated OH-PBDEs, after 45 and $90 \mathrm{~min}$ for 2'-OH-BDE66 and 2'-OH-BDE68, and after 45, 90, and $180 \mathrm{~min}$ for 6-OH-BDE47 and 6'-OH-BDE49. Increasing the amount of acid in the beginning of the reaction was avoided, to avoid high pressure build up in the Eppendorf tubes.

\section{Degradation of OH-PBDEs in sediment}

A standard mixture composed of 6-OH-BDE90, 6-OH-BDE99, 2-OH-BDE123, and 6-OH-BDE85, $100 \mathrm{ng}$ (170 pmol) each in 
$100 \mu \mathrm{L}$ of methanol, was spiked into a sterile test tube. The methanol was evaporated of until only a droplet remained before adding $1 \mathrm{~g}$ of sediment and $4 \mathrm{~mL}$ of filtered seawater. The ratio of sediment to water was the same as in the study conducted by Zhang and co-workers (2012). The test tubes, 27 in total, were placed on a tipping board during $48 \mathrm{~h}$ to achieve equilibrium. Three samples were then analyzed to determine the starting concentrations of OH-PBDEs and MeO-PBDEs in the sediment. The remaining samples were stored in darkness, at either room temperature or at $4{ }^{\circ} \mathrm{C}$. Three separate samples were analyzed per time point. The samples stored at room temperature were taken for analyses after 7, 30, 90, and 180 days respectively (in total 12 samples) while the samples stored at $4{ }^{\circ} \mathrm{C}$ were taken for analyses after 7, 30, 180, and 360 days respectively (in total 12 samples). Before analysis, all samples were centrifuged at $2000 \mathrm{~g}$ for $5 \mathrm{~min}$ and the water was decanted off. 4'-OH-BDE121 (50 ng) and BDE139 (5 ng) were added as surrogate internal standards for phenolic and neutral compounds, respectively.

\section{Determination of native levels of OH-PBDEs}

Two $5 \mathrm{~g}$ samples of sediment were analyzed to quantify the native occurrence of OH-PBDEs and MeO-PBDEs. 4'-OHBDE121 (5 ng) and BDE139 (1 ng) were added as internal standards for phenolic and neutral compounds, respectively, before starting the extraction. BDE139 (5 ng) was also added as volumetric standard in the phenolic fraction prior to instrumental analysis to calculate the recovery of the surrogate. A procedural blank sample was run in parallel to the samples, and instrumental blanks were run before and after all analyses; all analytes were below the limit of quantification (LOQ; 5 times the level of the surrounding noise) in the blanks. Only trace amounts of 6-OHBDE47 and 6-OH-BDE85 could tentatively be detected in the instrumental blanks, but not in any levels that could influence the results; hence, no blank subtraction was needed.

\section{Extraction of sediment}

The extractions were performed using a slightly modified version of the procedure described by Lindqvist and Asplund (2019). Briefly, to $1 \mathrm{~g}$ of sediment acetonitrile/2-propanol ( $3: 1,4 \mathrm{~mL}$ ) with $1 \%$ acetic acid was added before mixing the sample on a vortex mixer. The sample was then mixed again following the addition of diethyl ether (DEE; $2 \mathrm{~mL}$ ) before centrifuging the sample at $2000 \mathrm{~g}$ for $5 \mathrm{~min}$. The supernatant was transferred to a new test tube, and the sediment pellet was re-extracted with 2-methylpentane/diethyl ether (IHX/DEE; $3: 1,3 \mathrm{~mL}$ ). The sample was centrifuged and the supernatants from the two extractions combined. $\mathrm{HCl}$ $(6 \mathrm{M}, 0.5 \mathrm{~mL})$, water $(4 \mathrm{~mL})$, and IHX $(3 \mathrm{~mL})$ were added to the extract. After mixing and centrifugation, the organic phase was transferred to a new test tube (for the $5 \mathrm{~g}$ samples the extraction method was scaled up accordingly). The volume was reduced to approximately $4 \mathrm{~mL}$ under a stream of nitrogen before adding potassium hydroxide $(0.5 \mathrm{M}, 3 \mathrm{~mL})$ in water/ethanol $(1: 1)$. After mixing and centrifugation, the aqueous phase (phenolic fraction) and organic phase (neutral fraction) were transferred to separate test tubes.

The phenolic fraction was acidified with $\mathrm{HCl}(6 \mathrm{M}$, $0.5 \mathrm{~mL}$ ) and the phenolic compounds were back-extracted into IHX $(3 \mathrm{~mL})$. The aqueous phase was re-extracted with an additional portion of IHX $(1 \mathrm{~mL})$. After combining the organic phases, the volume was reduced to $0.5 \mathrm{~mL}$ and a few drops of methanol were added. Derivatization was performed by adding ethereal diazomethane $(300 \mu \mathrm{L})$. The reaction was allowed to proceed for $1.5 \mathrm{~h}$ at ambient temperature in darkness before the excess diazomethane was evaporated off under a gentle stream of nitrogen gas. After adjusting the volume to $3 \mathrm{~mL}$ with IHX, concentrated sulfuric acid $(1.5 \mathrm{~mL})$ was added. The sample was mixed and centrifuged before the IHX was transferred to a new test tube. The sulfuric acid was re-extracted with 2,2,4-trimethylpentane (TMP; $1 \mathrm{~mL}$ ).

The neutral fraction was reduced to $3 \mathrm{~mL}$ and treated with sulfuric acid in the same manner as the phenolic fraction. The neutral fraction was then further reduced to $2 \mathrm{~mL}$, and sulfur was removed by shaking the sample (in a closed test tube) under a tap of streaming hot water for $1 \mathrm{~min}$ with 2-propanol $(2 \mathrm{~mL})$ and a tetrabutylammonium hydrogen sulfate aqueous solution (4\% $\mathrm{w} / \mathrm{w})$ saturated with sodium sulfite $(2 \mathrm{~mL})$, according to Nylund and co-workers (1992). Water (5 mL) and TMP $(1 \mathrm{~mL})$ was added to the sample, and after phase separation by centrifugation, the organic phase was isolated. Both the neutral and the phenolic fractions were reduced by nitrogen gas until only TMP remained, and the volumes were further adjusted to proper levels before analysis on GC-MS.

\section{Instrumental analysis}

Analyses were conducted on an Agilent 7890A GC (Agilent Technologies, CA, USA) with a multimode injector (MMI), coupled to a 5975C MS. All analyses were done in the electron capture negative ionization mode (ECNI). A 5- $\mu \mathrm{L}$ aliquot was injected in liquid vent mode at $75^{\circ} \mathrm{C}$. The solvent was vented at $5 \mathrm{psi}$ for $0.04 \mathrm{~min}$. The injector was then ramped in two stages: first, to $255^{\circ} \mathrm{C}$ (held for $1.86 \mathrm{~min}$ ), and then, to $325^{\circ} \mathrm{C}$, both times at $600{ }^{\circ} \mathrm{C} / \mathrm{min}$. The split was opened after $2.2 \mathrm{~min}$ with a purge flow of $60 \mathrm{~mL} / \mathrm{min}$. Helium was used as carrier gas with a flow of $1.25 \mathrm{~mL} / \mathrm{min}$. The $\mathrm{GC}$ oven was programmed from $65{ }^{\circ} \mathrm{C}$ (held for $2.2 \mathrm{~min}$ ) to $250{ }^{\circ} \mathrm{C}$ at $25^{\circ} \mathrm{C} / \mathrm{min}$, then to 285 at $5{ }^{\circ} \mathrm{C} / \mathrm{min}$, and finally to $310^{\circ} \mathrm{C}$ at $25^{\circ} \mathrm{C} / \mathrm{min}$ (held for $5.4 \mathrm{~min}$ ). The whole program was $23 \mathrm{~min}$. A J\&W DB-35MS UI capillary column was used $(20 \mathrm{~m} \times$ $0.18 \mathrm{~mm}$ i.d. $\times 0.18 \mu \mathrm{m}$ film thickness; J\&W Scientific, CA, USA). The transfer line to the MS was set at $310{ }^{\circ} \mathrm{C}$, the ion source temperature was $200{ }^{\circ} \mathrm{C}$, and the quadrupole temperature was $150{ }^{\circ} \mathrm{C}$. Methane was used as buffer gas for 
ECNI/MS, with a vacuum pressure of $\sim 1.9 \mathrm{E}-04$ Torr. Quantifications of OH-PBDEs were conducted using selected ion monitoring (SIM) of the bromide ions $m / z$ : 79, 81. All quantifications were conducted using 5-point external calibration curves with authentic reference standards.

\section{Result and discussion}

\section{Chemical reductive debromination}

During chemical debromination, all test compounds degraded according to first-order kinetics (see example in Fig. 1). The rate of debromination was affected both by the level of oxidation (amount of bromines) as well as the substitution pattern, particularly by the occurrence of sterically congested bromines (i.e., bromines with two adjacent bulky substituents, e.g., $\mathrm{Br} / \mathrm{Br}$ or $\mathrm{Br} / \mathrm{OH})$. The difference in half-lives $\left(t_{1 / 2}\right)$ between the pentabrominated congeners and the two slowest degrading tetrabrominated congeners $(6-\mathrm{OH}-\mathrm{BDE} 47$ and 6'OH-BDE49) was as large as 2 orders of magnitude (see Table 1). In fact, 6-OH-BDE47 and 6'-OH-BDE49 did not reach $t_{1 / 2}$ within the time span of the experiment and their recorded $t_{1 / 2}$ values in Table 1 should only be considered tentative.

The effect of sterically congested bromines on the reduction rate can be observed by comparing the $t_{1 / 2}$ for the different tetrabrominated OH-PBDEs in Table 1. The only tetrabrominated OH-PBDE in Table 1 that has a sterically congested bromine is 2'-OH-BDE66, and this congener degraded 5 times faster than the second fastest degrading tetrabrominated congener. No statistically significant difference could be observed between 6-OH-BDE99 and 6MeOBDE99 in this system, while both showed statistical difference compared to BDE99 (using 2 sided $t$-tests, $p<0.05$ ). BDE99 may differ from its methoxylated and hydroxylated
Table 1 Chemical debromination rates provided as $t_{1 / 2}(\mathrm{~min}) \pm$ one standard deviation. All experiments were made in triplicates

\begin{tabular}{lllll}
\hline $\mathrm{Br}_{n}$ & Congener & $t_{1 / 2}(\mathrm{~min})$ & & \\
\hline 6 & 6-OH-BDE137 & 3.1 & \pm & 0.06 \\
5 & 6-OH-BDE85 & 3.5 & \pm & 0.5 \\
5 & 2-OH-BDE123 & 3.6 & \pm & 0.5 \\
5 & 6-OH-BDE90 & 4.6 & \pm & 0.6 \\
5 & 6-OH-BDE99 & 5.1 & \pm & 0.7 \\
5 & 6-MeO-BDE99 & 4.4 & \pm & 0.2 \\
5 & BDE99 & 9.7 & \pm & 0.3 \\
4 & 2'-OH-BDE66 & 23 & \pm & 3.0 \\
4 & 2'-OH-BDE68 & 122 & \pm & 14 \\
4 & 6'-OH-BDE49 & $364^{\mathrm{A}}$ & \pm & 75 \\
4 & 6-OH-BDE47 & $417^{\mathrm{A}}$ & \pm & 28 \\
\hline
\end{tabular}

${ }^{\mathrm{A}}$ These values should be considered approximations

counterparts both because it is less oxidized (it lacks the additional oxygen from the $\mathrm{MeO} / \mathrm{OH}$ group) and because it lacks sterically congested bromines.

The inclination of sterically congested bromines to be removed first can also be observed in the products formed when degrading the pentabrominated OH-PBDEs. 6-OH-BDE90 and 6-OH-BDE99 have one sterically congested bromine each (in position 2 and 5, respectively, see Fig. 2), and both degrade to form one major product by loss of their, respectively, sterically congested bromine. 6-OH-BDE90 form 2'-OH-BDE68, and 6-OH-BDE99 form 6-OH-BDE47. Both congeners also form 6'-OH-BDE49 as a moderate to minor product (via loss of the $\mathrm{Br}$ in position 3 and 4, respectively, see Fig. 2). 6-OHBDE99 also forms 2'-OH-BDE66 as well as an unknown congener as minor products (see Table S2 in the ESM). 6$\mathrm{OH}-\mathrm{BDE} 85$ and 2-OH-BDE123 on the other hand has two
Fig. 1 Example of the chemical reductive debromination of $\mathrm{OH}-$ PBDEs displaying the degradation for three congeners. Average values from three experiments. Full data set can be found in the electronic supplementary material (ESM), Table S1
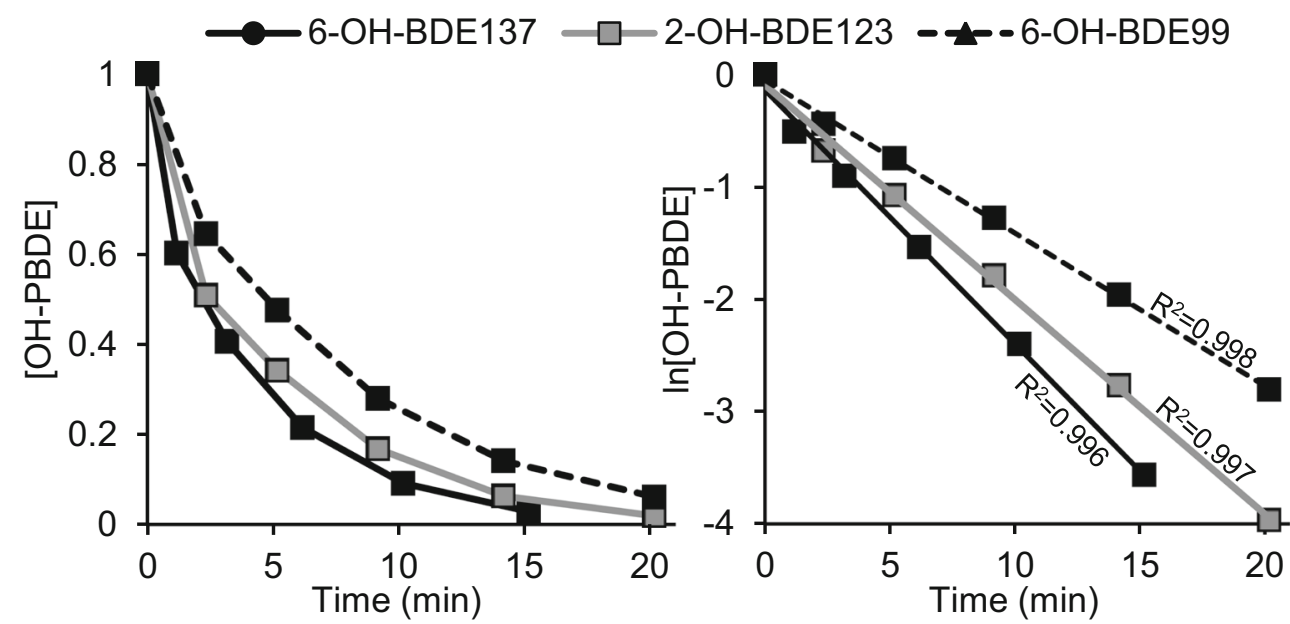
<smiles>Oc1cc(Br)c(Br)c(Br)c1Oc1ccc(Br)cc1Br</smiles>

6-OH-BDE90

6-OH-BDE85<smiles>Oc1c(Oc2ccc(Br)cc2Br)cc(Br)c(Br)c1Oc1cc(Br)cc(Br)c1Oc1ccc(Br)cc1Br</smiles>

6-OH-BDE99

2-OH-BDE123

Fig. 2 Chemical structures of the pentabrominated OH-PBDEs included in this study. The structures of all tetrabrominated OH-PBDEs that can be formed by removal of one $\mathrm{Br}$ from each of the four compounds can be found in the ESM, Fig. S1

sterically congested bromines (in position 2 and 3, respectively, 3 and 4, see Fig. 2) and hence form two major products. 6-OH-BDE85 form 6'-OH-BDE66 (by loss of Br in position 2) and 6-OH-BDE47 (by loss of $\mathrm{Br}$ in position 3), while 2-OH-BDE123 form 6'-OH-BDE66 (by loss of $\mathrm{Br}$ in position 3) and 2'-OH-BDE68 (by loss of $\mathrm{Br}$ in position 4) (see Fig. 2). Both also form minor products (see Table S2 in the ESM). The exact formation rate was not measured as the products themselves start to degrade at variable speed upon formation resulting in the formation of tribrominated congeners (see Table S2 in the ESM). The steric congestion likely leads to tension in the molecule due to unfavorable bond angles, which in turn results in a higher energy state. Removal of the sterically congested bromines would relieve the tension leading to the lowest energy product. The dominant formation of 6-OH-BDE47, 2'-OH-BDE68, and 6'-OH-BDE66 from the four pentabrominated congeners shown in Fig. 2 can thus be predicted by calculating the energies of the products formed, see Table S3 in the ESM.

The degradation rate of $6^{\prime}-\mathrm{OH}-\mathrm{BDE} 66$ was not measured due to the lack of a pure standard during the experiments $(6$ 'MeO-BDE66 was however available in a mix of MeO-PBDEs for identification of methylated 6'-OH-BDE66). The lack of sterically congested bromines in 6'-OH-BDE66 does however suggest that it at least would degrade slower than $2^{\prime}-\mathrm{OH}-$ BDE66. Only two tribrominated congener (6'-OH-BDE17 and 2'-OH-BDE28) were available as standards for positive identification among the products formed by debromination of the tetrabrominated OH-PBDEs. However, a full list of the different products that were detected, together with their respective retention time on the GC system used, can be found in the ESM, Table S2.

\section{Degradation of OH-PBDEs in sediment}

The four pentabrominated OH-PBDEs chosen for this experiment are the dominant pentabrominated congeners detected in Baltic Sea algae, as well as in other Baltic Sea organisms (Löfstrand 2011; Lindqvist 2016). All four OH-PBDEs were observed to degrade rapidly in the spiked sediment at room temperature (see Fig. 3). 6-OH-BDE90 and 6-OH-BDE99 degraded by more than $50 \%$ within the first 7 days (see Table S4 in the ESM). In direct contrast to the results from the chemical debromination, 6-OH-BDE90 and 6-OHBDE99 were observed to degrade faster than 2-OH-BDE123 and 6-OH-BDE85 in sediment. 2-OH-BDE123 degraded only slightly slower, while 6-OH-BDE85 degraded significantly slower than 6-OH-BDE90 and 6-OH-BDE99 (see Table S4 in the ESM).

The major debromination products formed also differed from that obtained during the chemical debromination

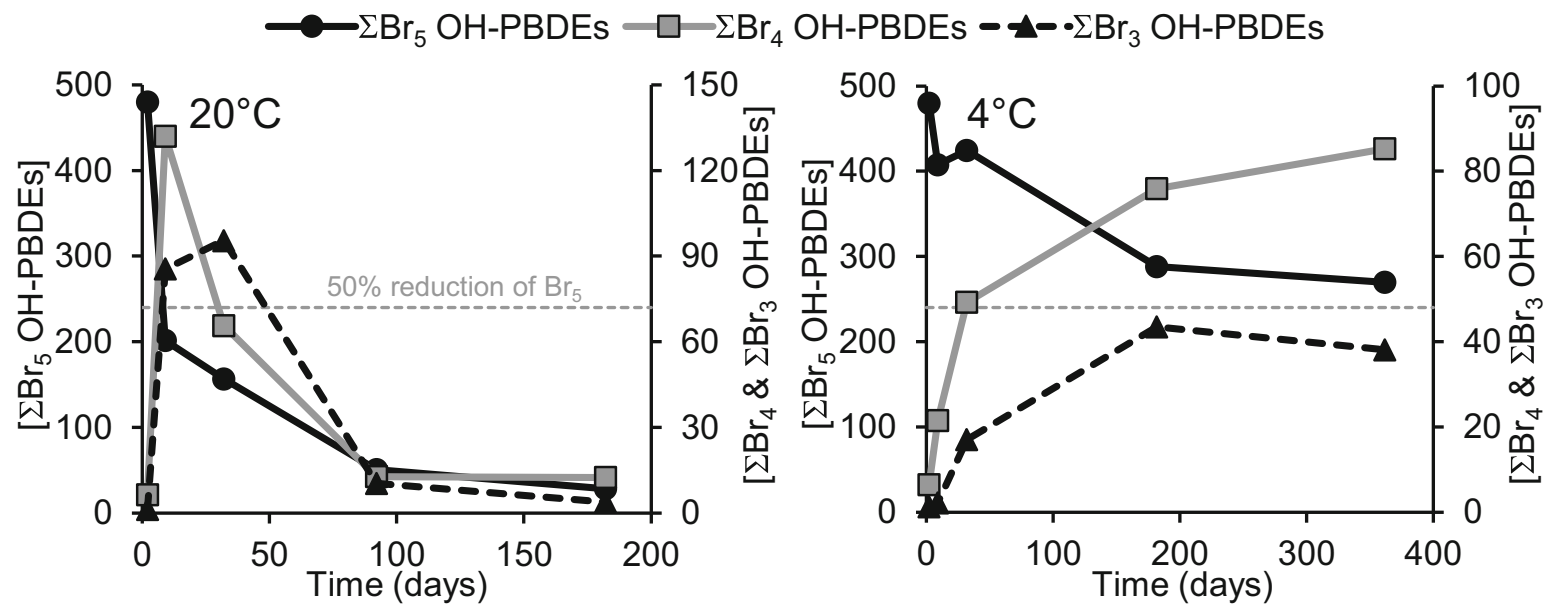

Fig. 3 Degradation of spiked pentabrominated OH-PBDEs in sediment and formation of the major tetra- and tribrominated congeners, at room temperature as well as at $4{ }^{\circ} \mathrm{C}$. Concentrations are provided in pmol/g wet weight. Raw data can be found in the ESM, Table S4 and S5 
experiment. The microbial debromination of the four pentabrominated OH-PBDEs in sediment initially yielded a quite large diversity of tetrabrominated congeners. In total, eight tetrabrominated products with significant yields were quantified, of which three were of unknown substitution pattern and were pseudo quantified against 2'-OH-BDE66 (the response factor between different tetrabrominated congeners differed only marginally on the GC-MS system used). The major tetrabrominated congener formed was 6'-OH-BDE49 followed by an unknown compound (U4-OH-A in Table S4, in the ESM). During chemical debromination, on the other hand, 6'-OH-BDE49 was only formed as a moderate to minor product from 6-OH-BDE90 and 6-OH-BDE99, while 2'-OHBDE68 and 6-OH-BDE47, respectively, were formed as the major products. The difference in the product composition gained between chemical debromination and debromination in sediment highlights the added selectivity during debromination in nature. Of course, the product composition may vary depending on several abiotic factors such as redox potential and $\mathrm{pH}$, as well as by the composition of bacteria strains in the sediment. The chemical debromination conducted in this study utilized hydrochloric acid, which means that the $\mathrm{pH}$ will be much lower than what is usually found in natural sediments.

As the debromination progressed further in the sediment, two tribrominated congeners became utterly dominant. One of these congeners was indicated as a minor product of $6^{\prime}-\mathrm{OH}-$ BDE49 in the chemical debromination experiment (U3-OHBDE49 in Table S4, in the ESM) and the other as a minor product of 6-OH-BDE47 (U3-OH-BDE47 in Table S4, in the ESM). However, the large increase of these two congeners in the sediment indicates that some of the unknown tetrabrominated congeners likely degrade to either of these two tribrominated congeners as well (see Table S4, in the ESM). Both these congeners were pseudo quantified against 6'-OH-BDE17. After 7 days in room temperature, the increase in concentration of tetra- and tribrominated congeners accounted for $75 \%$ of the decrease in concentration of the added pentabrominated congeners, which proves that debromination is the major degradation route for pentabrominated OH-PBDEs. However, after one month, even the tribrominated congeners started to decrease in concentration (see Fig. 3), while no significant formation of dibrominated congeners was observed. The lack of formed dibrominated congeners indicates that other degradation routes become more important for the less brominated congeners. Representative chromatograms from the experiment can be found in the ESM, Fig. S2.

At $4{ }^{\circ} \mathrm{C}$, no statistically significant degradation of the spiked pentabrominated OH-PBDEs could be determined until after 6 months, at which point $30-50 \%$ had been degraded, mainly by debromination (see Fig. 3 as well as Table S5 in the ESM). Only 6-OH-BDE99 reached 50\% degradation within
6 months (see Table S5 in the ESM). After one year, 6-OHBDE90 had reached $50 \%$ degradation as well, while $2-\mathrm{OH}-$ BDE123 and 6-OH-BDE85 had decreased in concentration by $39 \%$ and $32 \%$, respectively (see Table S5 in the ESM). The increase of the tetra- and tribrominated congeners was more easily measured and a significant increase of some congeners could be observed already after 7 days at $4{ }^{\circ} \mathrm{C}$ (see Fig. 3 as well as Table S5 in the ESM).

During sediment incubation experiments with 6-OHBDE47, using marine sediment from Liaodong Bay (China), Zhang and co-workers (2012) observed that most of the added compound had been biotransformed to 6-MeO-BDE47 after 66 days at $30{ }^{\circ} \mathrm{C}$. In the present study, methylation of $\mathrm{OH}-$ PBDEs was observed to be much slower than debromination (see Fig. 4 as well as Tables S6 and S7 in the ESM). After 6 months at room temperature, the increase of pentabrominated MeO-PBDEs corresponded to $1.5 \%$ of the decrease in pentabrominated OH-PBDEs (see Table S6 in the ESM). When adding up all the MeO-PBDEs formed after 6 months in room temperature, they accounted for $9.5 \%$ of the degraded pentabrominated OH-PBDEs (see Table S6 in the ESM). At $4{ }^{\circ} \mathrm{C}$, the methylation progressed even slower (see Table S7 in the ESM). The difference in the biotransformations observed between the two studies may be the result of a large difference in the bacterial flora between the marine sediment of Liaodong Bay and the brackish water sediment of the northern Baltic proper.

Although the degradation of OH-PBDEs progressed much slower at $4{ }^{\circ} \mathrm{C}$ compared to room temperature, it still seems as if Baltic Sea sediment may have a high capacity for degrading these compounds. The major initial route for degradation seems to be debromination. However, as the OH-PBDEs become less brominated, other degradation routes seem to take over. A small amount of methylation is to be expected in Baltic Sea sediment, although not to the extent as might be observed in, e.g., the marine sediment of Liadong Bay (Zhang et al. 2012). As can be observed in Fig. 4, after 6 months at room temperature, all the determined $\mathrm{OH}-\mathrm{PBDEs}$ and $\mathrm{MeO}-$

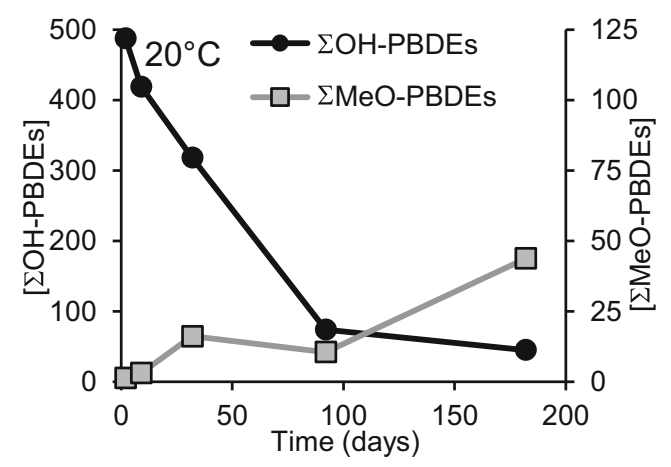

Fig. 4 Changes in $\Sigma \mathrm{OH}-\mathrm{PBDE}$, $\Sigma \mathrm{MeO}-\mathrm{PBDE}$ concentration over time at room temperature. Concentrations are provided in $\mathrm{pmol} / \mathrm{g}$ wet weight. Raw data can be found in the ESM Table S4 and S6 
Fig. 5 Native occurrence of $\mathrm{OH}-$ PBDEs and MeO-PBDEs in Baltic Sea surface sediment. Concentrations are provided in $\mathrm{pmol} / \mathrm{g}$ wet weight (w.w.). Raw data can be found in the ESM, Table S8

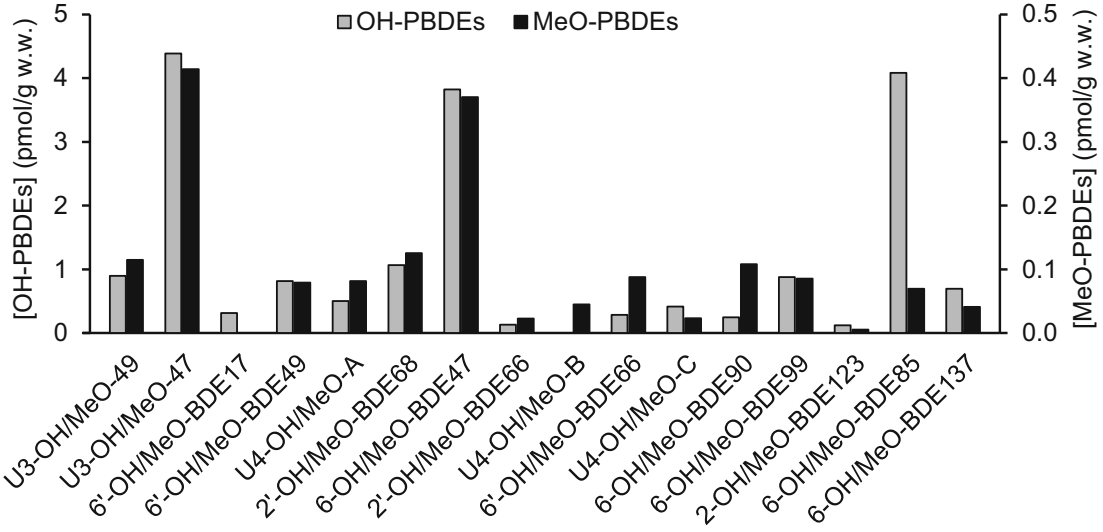

PBDEs together only accounted for $20 \%$ of the starting concentration, meaning that $80 \%$ had been degraded to something that could not be observed using the analytical method applied in this study. Chemical debromination could not be used to predict the degradation products in the sediment. In this study, only surface sediment was used, and it is likely that bacterial activity decreases deeper down in the sediment and thus that the biological degradation of OH-PBDEs slows down in Baltic Sea sediment over time.

\section{Native occurrence of $\mathrm{OH}$ - and MeO-PBDEs in sediment}

6-OH-BDE85 was by far the most abundant pentabrominated $\mathrm{OH}-\mathrm{PBDE}$ in the collected Baltic Sea surface sediment (see Fig. 5). The dominance of 6-OH-BDE85 in the sediment is likely a reflection of the large production of this congener observed in Baltic Sea macroalgae, e.g., C. tenuicorne (Lindqvist et al. 2017). 6-OH-BDE47, a potential degradation product of 6-OH-BDE85, was the dominant tetrabrominated congener, and its degradation product U3-OH-BDE47 was the dominant tribrominated congener. Among red, green, and brown filamentous macroalgae in the Baltic Sea the $\mathrm{OH}-$ PBDE congener pattern is normally dominated by $6-\mathrm{OH}-$ BDE85 and 6-OH-BDE137 shortly followed by 6-OHBDE47, while tribrominated congeners are rarely observed (Löfstrand 2011; Lindqvist et al. 2017). The levels of MeOPBDEs were about 10 times lower than that of the corresponding OH-PBDEs (see Fig. 5). Similar relationships between $\mathrm{OH}-$ and MeO-PBDEs have also been observed in algae, e.g., C. tenuicorne (Dahlgren et al. 2015). Considering the slow conversion from OH-PBDEs to MeO-PBDEs that was observed in the degradation experiment (see Fig. 4), in comparison to debromination (see Fig. 3), a general increase of $\mathrm{MeO}-\mathrm{PBDEs}$ deeper down in the sediment is not expected.
Acknowledgments Thanks to Göran Marsh for synthesizing most of the standards used in this study. Martin Ogonowski is acknowledged for his assistance in the sediment sampling.

Author contributions DL started the project, designed the study, and had the main responsibility for interpreting the data and writing the article. JG conducted sediment extractions and aided in quantifications, data handling, and writing of the article.

Funding Open Access funding provided by Stockholm University.

Data availability All relevant data are included in the supplementary material.

\section{Compliance with ethical standards}

Ethical approval No ethical approval was necessary for this study.

Consent to participate All participants in this study consent to participation.

Consent to publish All authors consent to this publication.

Competing interests The authors declare that they have no competing interests.

Open Access This article is licensed under a Creative Commons Attribution 4.0 International License, which permits use, sharing, adaptation, distribution and reproduction in any medium or format, as long as you give appropriate credit to the original author(s) and the source, provide a link to the Creative Commons licence, and indicate if changes were made. The images or other third party material in this article are included in the article's Creative Commons licence, unless indicated otherwise in a credit line to the material. If material is not included in the article's Creative Commons licence and your intended use is not permitted by statutory regulation or exceeds the permitted use, you will need to obtain permission directly from the copyright holder. To view a copy of this licence, visit http://creativecommons.org/licenses/by/4.0/. 


\section{References}

Dahlgren E, Enhus C, Lindqvist D, Eklund B, Asplund L (2015) Induced production of brominated aromatic compounds in the alga Ceramium tenuicorne. Environ Sci Pollut Res 22:18107-18114. https://doi.org/10.1007/s11356-015-4907-7

Gribble G (2010) Naturally occurring organohalogen compounds - a comprehensive update. Prog Chem Org Nat Prod 91. https://doi. org/10.1007/978-3-211-99323-1

HELCOM (2009) Eutrophication in the Baltic Sea: an integrated thematic assessment of the effects of nutrient enrichment in the Baltic Sea region. Helsinki Commission, Baltic Marine Environment Protection Commission https://helcom.fi/media/publications/ BSEP115B-1.pdf.

Isaacson PJ, Frink CR (1984) Nonreversible sorption of phenolic compounds by sediment fractions: the role of sediment organic matter. Environ Sci Technol 18:43-48. https://doi.org/10.1021/ es00119a010

Lindqvist D (2016) Hydroxylated polybrominated diphenyl ethers in Baltic Sea biota - natural production, food we distribution and biotransformation. Stockholm University, Disertation

Lindqvist D, Dahlgren E, Asplund L (2017) Biosynthesis of hydroxylated polybrominated diphenyl ethers and the correlation with photosynthetic pigments in the red alga Ceramium tenuicorne. Phytochemistry 133:51-58. https://doi.org/10.1016/j.phytochem. 2016.10.009

Lindqvist D, Asplund L (2019) Determination of hydroxylated polybrominated diphenyl ethers in blood from Baltic grey seals. J Anal Test 3:287-294. https://doi.org/10.1007/s41664-019-00084-1

Löfstrand K (2011) Trends and exposure of naturally produced brominated substances in Baltic biota - with focus on OH-PBDEs and PBDDs. Stockholm University, Disertation

Löfstrand K, Liu X, Lindqvist D, Jensen S, Asplund L (2011) Seasonal variations of hydroxylated and methoxylated brominated diphenyl ethers in blue mussels from the Baltic Sea. Chemosphere 84:527532. https://doi.org/10.1016/j.chemosphere.2011.01.001

Marsh G, Stenutz R, Bergman $\AA$ (2003) Synthesis of hydroxylated and methoxylated polybrominated diphenyl ethers - natural products and potential polybrominated diphenyl ether metabolites. European J Org Chem 2003:2566-2576. https://doi.org/10.1002/ ejoc. 200300081

Nylund K, Asplund L, Jansson B, Jonsson P, Litzén K, Sellström U (1992) Analysis of some polyhalogenated organic pollutants in sediment and sewage sludge. Chemosphere 24:1721-1730. https://doi. org/10.1016/0045-6535(92)90227-I

Schellenberg K, Leuenberger C, Schwarzenbach RP (1984) Sorption of chlorinated phenols by natural sediments and aquifer materials. Environ Sci Technol 18:652-657. https://doi.org/10.1021/ es00127a005

Tashiro M, Fukata G, Fukata G (1976) The reductive debromination of bromophenols. Org Prep Proced Int 8:231-236. https://doi.org/10. 1080/00304947609355631

Tokarz JA, Ahn MY, Leng J, Filley TR, Nies L (2008) Reductive debromination of polybrominated diphenyl ethers in anaerobic sediment and a biomimetic system. Environ Sci Technol 42:11571164. https://doi.org/10.1021/es071989t

Wan Y, Wiseman S, Chang H, Zhang X, Jones PD, Hecker M, Kannan K, Tanabe S, Hu J, Lam MHW, Giesy JP (2009) Origin of hydroxylated brominated diphenyl ethers: natural compounds or man-made flame retardants? Environ Sci Technol 43:7536-7542. https://doi. org/10.1021/es $901357 \mathrm{u}$

Zhang K, Wan Y, Jones PD, Wiseman S, Giesy JP, Hu J (2012) Occurrences and fates of hydroxylated polybrominated diphenyl ethers in marine sediments in relation to trophodynamics. Environ Sci Technol 46:2148-2155. https://doi.org/10.1021/es203195s

Publisher's note Springer Nature remains neutral with regard to jurisdictional claims in published maps and institutional affiliations. 